\title{
Possibilités d'infestation par les pucerons : Cedrobium laportei Remaudière, Cinara cedri Mimeur (Homoptera, Lachnidae), chez le genre Cedrus
}

\author{
J.P. FABRE \\ Avec la collaboration technique de A. CHALON, J. CHIZKY, E. ROBERT \\ INRA, Station de Zoologie forestière \\ Avenue Vivaldi, F 84000 Avignon
}

\section{Résumé}

Cette note apporte des précisions sur les possibilités d'infestation par deux pucerons: Cedrobium laportei Remaudière, Cinara cedri Mimeur, sur : Cedrus atlantica Manetti, $C$. libani Loudon, C. brevifolia Henry, C. deodara Loudon.

Dans 2 plantations, nous avons comparé les densité d'infestation spontanée des deux pucerons. C. cedri attaque les 4 espèces de cèdre. $C$. laportei infeste aussi $C$. atlantica et $C$. deodara, mais $C$. brevifolia et $C$. libani étaient indemnes.

Nous avons effectué des infestations artificielles de $C$. laportei. Le cèdre de l'Atlas est toujours l'espèce la plus infestée, $C$. deodara l'est également, mais la fixation de ce puceron est possible sur C. libani et sur C. brevifolia.

Des différences de densités d'infestation naturelle ont été mises en évidence, pour les deux pucerons, sur certaines provenances.

Mots clés: Cèdres, Cedrus atlantica, Cedrus libani, Cedrus brevifolia, Cedrus deodara. Homoptera, Lachnidae, Cedrobium laportei, Cinara cedri, résistance naturelle.

\section{Introduction}

Le genre Cedrus comprend 4 espèces assez bien caractérisées par leur morphologie (Arbez et al., 1978), leur biologie (Toтн, 1982-1984), leur écologie (AusSENAC, 1983). Leurs aires naturelles de répartition ne se recouvrent pas. C. atlantica Manetti occupe certaines montagnes d'Afrique du Nord, principalement celles du Maroc (AchHAL et al., 1980) : Rif, Moyen-Atlas, partie orientale du Haut-Atlas ; en Algérie, son aire est plus morcelée, les peuplements les plus importants étant situés dans les Aurès. C. libani Loudon et $C$. brevifolia Henry le remplacent dans la partie orientale du bassin méditerranéen (QUEZEL \& BARBERo, 1985) : le premier se rencontre sur le versant occidental de la chaîne libanaise (Beals, 1955 ; ABI-SALEH, 1978), où ne subsistent que quelques petits peuplements isolés, un peu en Syrie, mais surtout en Turquie, dans les montagnes du Taurus et de l'Anti-Taurus (Quezel \& Pamukcuogle, 1973) ; l'aire du 
second se limite aux montagnes situées au sud-ouest de l'île de Chypre. Enfin, $C$. deodara Loudon s'étend sur l'Afghanistan jusqu'au Népal occidental.

Ces quatre espèces sont aujourd'hui présentes en Europe, où elles ont été introduites comme essences de reboisement ou comme arbres d'ornement.

Deux espèces de pucerons, elles aussi présentes aujourd'hui sur le continent européen, sont inféodées au genre Cedrus :

- La première, Cedrobium laportei Rem., a été observée tout d'abord au Maroc, dès 1935, par Mimeur, qui l'a confondue avec l'espèce suivante (Remaudière, 1954), puis ensuite en Algérie (Remaudière, 1954), en Espagne (F. RoBredo, communication personnelle), en France (Emonnot et al, 1967 ; Fabre, 1982 ; Fabre \& Rabasse, 1987), en Italie (Covassi, 1971; Roberti, 1976), en Angleterre (CArTer \& MASlen, 1982), aux Pays-Bas (Rossem et al., 1976). Dans la majorité des cas, elle est signalée sur $C$. atlantica ou sur certaines de ses variétés ornementales (var. glauca, var. argentea), parfois sur "Cedrus sp. ». Covassi (1971) et RoberTi (1976) l'ont observée en Italie sur C. libani. En revanche, elle n'a pas été trouvée sur cette essence en Turquie ni en Syrie, ni sur C. deodara dans l'est de l'Afghanistan (Emonnot et al., 1967), et n'est pas signalée sur $C$. brevifolia.

- La seconde espèce : Cinara cedri Mimeur est connue au Maroc (Mimeur, 1935), en Espagne (Notario et al., 1978), en France (FABre, 1976), en Italie (Covassi \& Binazzi, 1974), en Turquie (Tuntay \& Remaudière, 1964). Elle a également été récoltée au Liban dans la Forêt de Barouk en 1980 par J. Riom (communication personnelle). Les plantes hôtes mentionnées par les auteurs sont: Cedrus sp., $C$. atlantica, C. atlantica var. glauca, C. libani, C. deodara, C. deodara var. pendula. Comme la précédente, elle n'est pas signalée sur $C$. brevifolia.

L'objet de cette étude préliminaire est de préciser les possibilités d'infestation des 2 pucerons sur les 4 espèces de cèdres. L'intérêt de ce type d'étude est double : scientifique, d'une part, car ce peut être un point de départ d'analyses plus approfondies des relations cèdres-pucerons; pratique, d'autre part, pour une extension plus rationnelle de l'aire de $C$. atlantica, essence très utilisée en reboisement dans le sud de la France.

\section{Matériel et méthodes}

Ce travail a été réalisé, pour l'essentiel, dans des plantations situées dans le sud-est de la France. Nous y avons procédé, d'une part, à une évaluation de l'importance d'infestations spontanées de pucerons, d'autre part (seulement pour C. laportei), au suivi d'infestations artificielles.

\subsection{Evaluation des infestations naturelles de pucerons}

Les observations ont été réalisées en juin 1980, juin et octobre 1981 et juin 1985 (un relevé chaque fois), dans deux plantations comparatives situées dans le Var, la première en Forêt domaniale de Collobrières, au lieu-dit Le Treps, la seconde, en Forêt domaniale de Pellenc. Ces plantations ont été mises en place à partir de 1972 par la station INRA d'Amélioration des arbres forestiers (ANONYME, 1975 ; ARBEz et al., 
1978 ; Pradal, 1979 ; Veyret, 1984). Dans les deux cas, nous avons examiné plusieurs groupes de 6 à 25 arbres. Pour chaque groupe, les arbres ont été choisis au centre de 2 à 3 parcelles où les différentes provenances composant la plantatín se trouvaient représentées. Malheureusement, l'échantillon ne permettait pas d'exploiter toutes les possibilités offertes par les dispositifs expérimentaux : lattices équilibrés à Pellenc, blocs incomplets équilibrés au Treps. La vitesse de multiplication des pucerons, leur disparition tout aussi brutale interdit de poursuivre le même relevé après le $4^{\mathrm{e}}$ ou le $5^{\mathrm{c}}$ jour. Les données ont donc été exploitées par la méthode de l'analyse de la variance à 1 facteur contrôlé à partir des données individuelles. Lors de chaque relevé, la densité des deux espèces de pucerons sur chaque arbre a été déterminée de la façon suivante:

Muni d'un chronomètre, l'observateur examine à l'œeil nu l'ensemble des rameaux de l'arbre, à la recherche des colonies de pucerons. Celles de C. laportei sont localisées à la face inférieure des rameaux, essentiellement sur les entre-nœuds des pousses aoûtées de l'année précédente ; on rencontre aussi des individus à l'aisselle des aiguilles des pousses turgescentes de l'année. $C$. cedri se trouve sur les rameaux aoûtés des années précédentes ou sur le tronc. Les deux espèces peuvent se mélanger, mais les larves de $C$. cedri sont de plus grande taille. De plus, leurs soies dorsales sont dépourvues de renflement apical.

Chaque colonie est dénombrée, en distinguant, d'une part les larves, tous stades confondus, et d'autre part les ailés. Ces deux catégories seront ici regroupées. Soit alors $s_{\mathrm{i}}$ le nombre total des individus de l'un des deux pucerons portés sur l'arbre i et $t_{\mathrm{i}}$ le temps (en minutes) pris par l'examen de cet arbre. L'utilisation directe des valeurs $s_{i}$ ne tiendrait pas compte, ni de la variabilité individuelle de la taille des arbres, ni de la variabilité entre provenances, souvent élevée, de leur taille moyenne. Aussi les pondèrons-nous par $t_{i}$, en calculant la densité suivante :

$$
d_{\mathrm{i}}=10 * s_{\mathrm{i}} / t_{\mathrm{i}}
$$

qui est la valeur de $s_{\mathrm{i}}$ rapportée à un arbre «standard ", dont l'examen demanderait 10 minutes. Cette transformation, déjà utilisée par Shands \& Simpson (1955), suppose que l'observateur travaille toujours à la même vitesse ; autrement dit, on admet que la longueur totale des extrêmités de rameaux observées, qu'on devrait mesurer pour pouvoir calculer la densité réelle des pucerons, est proportionnelle au temps nécessaire pour effectuer leur examen, les densités moyennes respectives des deux pucerons par arbre sont ensuite calculées pour chacune des provenances puis comparées à l'aide du test de Duncan.

\subsection{Infestations artificielles}

Cette technique n'a été utilisée que pour $C$. laportei et visait à vérifier la "résistance " à cette espèce de certaines provenances. Les infestations ont été effectuées de 1983 à 1985 (cf. tabl. 1), certaines en serre, d'autres dans de petites plantations comparatives installées dans le Vaucluse, sur le terrain de la Station de Recherches forestières d'Avignon et à $15 \mathrm{~km}$ de là, sur la commune des Vignères. Une expérience du même type a également été effectuée en 1985 dans la plantation comparative de Pellenc.

Les colonies de $C$. laportei ont été prélevées parfois sur place, le plus souvent dans d'autres localités, ou encore dans une serre où nous disposons d'un élevage permanent. Les arbres infestés par le puceron sont tout d'abord repérés grâce aux fourmis qui circulent sur leur tronc. Puis les rameaux portant des colonies, sont coupés au sécateur, 


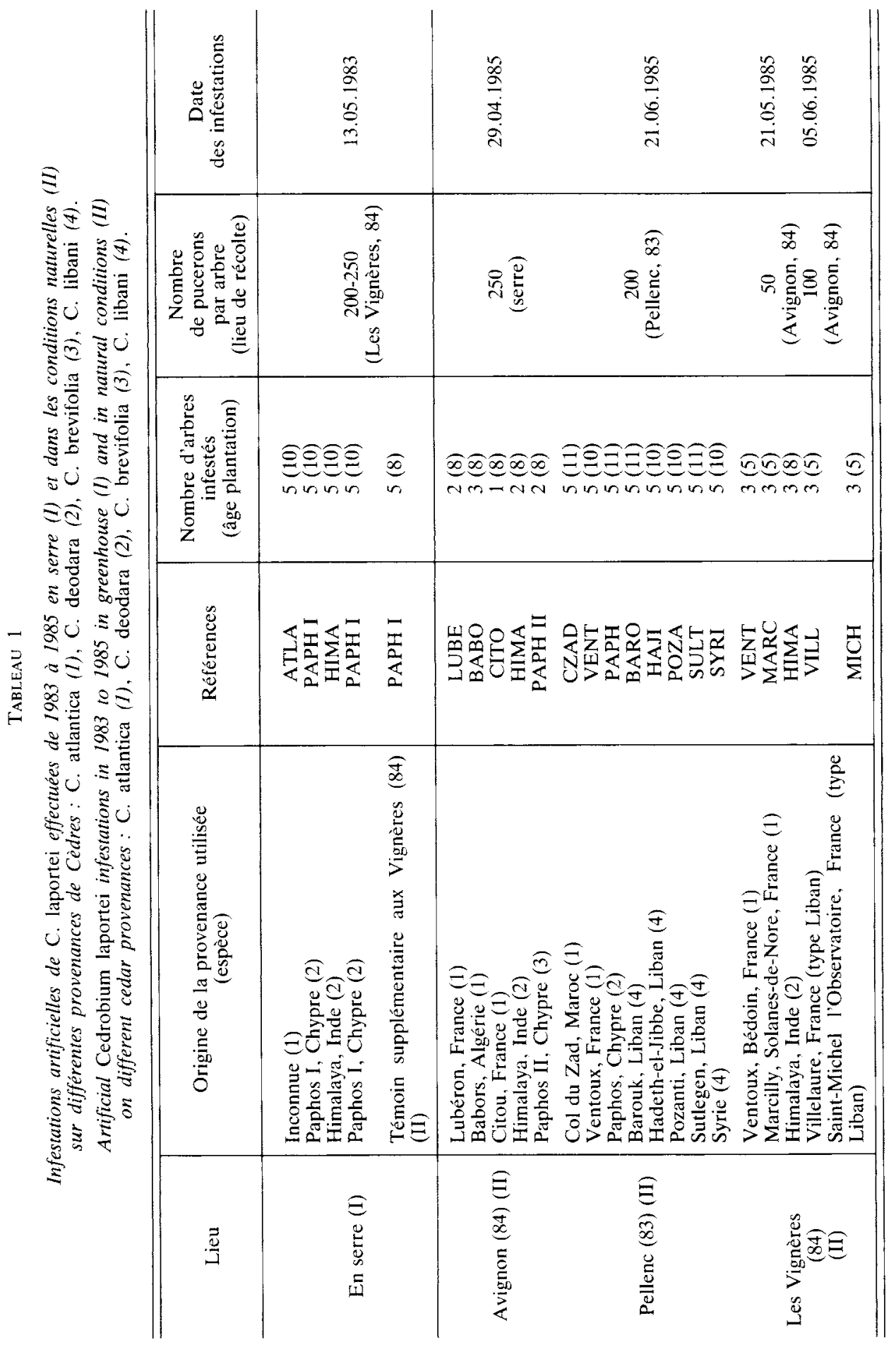


et placés immédiatement dans une glacière où règne une température d'environ 5 " $\mathrm{C}$. De cette façon, et à condition que la durée du transport ne dépasse pas 4 à 5 heures, les pucerons restent fixés à leur sụpport jusqu'au moment de l'infestation. Les rameaux sont alors sortis de la glacière et sectionnés en tronçons dont la longueur est fonction du nombre de pucerons désiré. Puis chaque tronçon est fixé, à l'aide d'un "plastilien », sur l'extrémité d'un rameau de l'arbre à infester. 'Généralement, on dispose ensuite autour de ce rameau un manchon de tissu de coton attaché à ses deux extrémités. Ce manchon permet de retrouver facilement les individus qui meurent sans s'être fixés. II protège aussi les nouvelles colonies de l'arrivée des prédateurs et de l'action néfaste des gros orages. Trois jours après l'infestation, on dénombre les individus qui se sont fixés sur les extrémités des rameaux et ceux qui sont morts. Les relevés suivants sont espacés d'environ une semaine. Ils sont poursuivis jusqu'à la disparition complète de tous les pucerons sur tous les arbres.

\section{Résultats et discussion}

\subsection{Infestations spontanées de pucerons}

Les tableaux 2 et 3 donnent les densités moyennes respectives des 2 pucerons dans chacune des 2 plantations et. pour chacune des origines géographiques de cèdres étudiées.

\subsection{Cinara cedri}

Au Treps, au mois de juin 1985, toutes les origines géographiques choisies ont été infestées par cette espèce. Par contre, elle n'a pas été trouvée sur $C$. atlantica originaire du Grand-Atlas au mois de juin 1980, sur toutes les provenances au mois de juin 1981 et sur la provenance artificielle française du Rialsesse au mois d'octobre 1981. En 1980, la densité moyenne du puceron par arbre atteignait pourtant $62,5 \pm 47,3(36,8 \pm 14,2$ en 1985), mais les colonies s'étaient développées sur 14 arbres seulement, les autres (186) étant indemnes. En juin 1981, au Treps, aucun individu n'a été découvert, la population ayant quasiment disparu, sans doute suite à l'action des prédateurs : coccinelles, chrysopes encore présents sur les arbres. Enfin, en octobre de la même année, la densité d'infestation était trop faible : $0,2 \pm 0,2$.

A Pellenc, toutes les origines géographiques ont été infestées soit au mois de juin 1985, soit au mois de juin 1981. Son absence sur 2 provenances en 1981 était due là aussi à la faible densité d'infestation (5,2 \pm 3,6 pucerons par arbre).

En 1981, au Treps comme à Pellenc, la densité d'infestation moyenne entre les provenances de cèdres étant respectivement de 0,6 , aucune différence significative entre elles n'a pu être mise en évidence. En 1980 au Treps, la densité d'infestation était plus élevée : 57 pucerons par arbre et par provenance, mais les colonies s'étaient développées sur certains arbres (coefficient de variation : 4,5 sur les 6 provenances du Moyen-Atlas). En revanche, à Pellenc en 1985, excepté la provenance HAJI, les autres de l'espèce libani étaient moins infestées (tabl. 4). 


\begin{tabular}{|c|c|c|c|c|c|c|c|c|c|}
\hline$\dot{\vec{B}}_{\dot{\overrightarrow{3}}}$ & \multirow{3}{*}{ 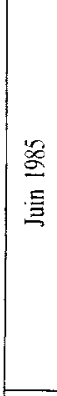 } & \multirow{2}{*}{ 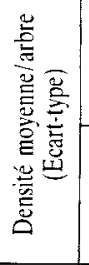 } & 离 & 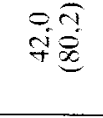 & 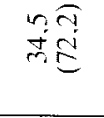 & $\begin{array}{l}r \sqrt{n} \\
\therefore 8 \\
\Rightarrow 8\end{array}$ & $\begin{array}{l}\infty \hat{\tilde{g}} \\
\infty \\
\infty\end{array}$ & 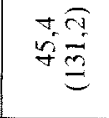 & 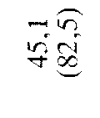 \\
\hline 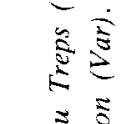 & & & 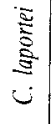 & 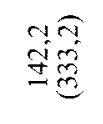 & 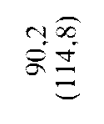 & $=1$ & 01 & $g \widehat{\vec{b}}$ & 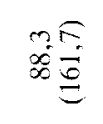 \\
\hline 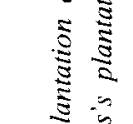 & & 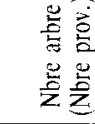 & & $0 \widehat{\Xi}$ & d] & $=\overparen{\Xi}$ & $\mathrm{y}=$ & $\infty$ & $\Xi \Xi$ \\
\hline 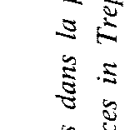 & & 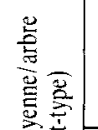 & $\begin{array}{c}\tilde{z} \\
\tilde{u} \\
ن\end{array}$ & $\begin{array}{l}\mathscr{\infty} \\
= \\
=\end{array}$ & बैత & $9=$ & $\begin{array}{l}\forall \propto \alpha \\
0 \\
0\end{array}$ & | 1 & 01 \\
\hline 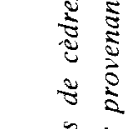 & 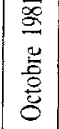 & 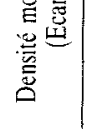 & 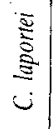 & $\infty \Xi$ & "ृ气 & 01 & $=1$ & 11 & 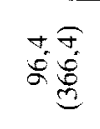 \\
\hline 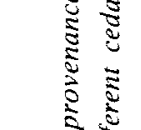 & & 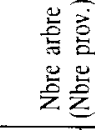 & & $\underline{\Xi}$ & 20 & $\vec{\nabla} \Xi$ & 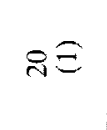 & 11 & 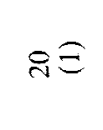 \\
\hline 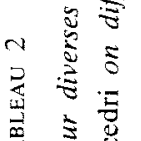 & & 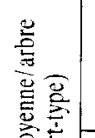 & $\stackrel{\Xi}{\mathrm{s}}$ & $=1$ & 01 & $=1$ & 01 & 11 & $=1$ \\
\hline 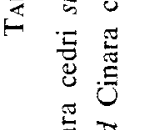 & 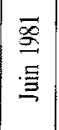 & 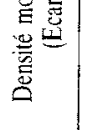 & 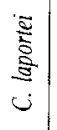 & 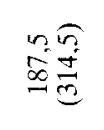 & 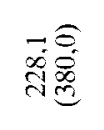 & $=1$ & 01 & 11 & 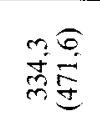 \\
\hline 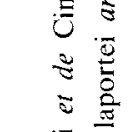 & & 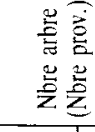 & & 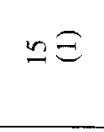 & $8 \sqrt{2}$ & $\sqrt{3} \cong$ & 요 & 11 & $\stackrel{i}{ }=$ \\
\hline 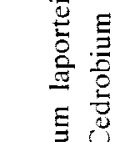 & & 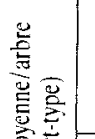 & $\begin{array}{l}\frac{\pi}{2} \\
\mathrm{U} \\
\mathrm{U}\end{array}$ & 01 & $\begin{array}{l}0 \hat{\sigma} \\
\hat{\sigma} \tilde{y}\end{array}$ & बid & $\stackrel{=}{\circ}$ & 11 & $+\stackrel{\overparen{g}}{\Xi}$ \\
\hline 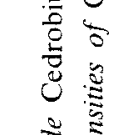 & $\begin{array}{l}\text { 产 } \\
\text { 高 }\end{array}$ & 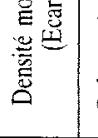 & 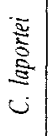 & 总 & $\begin{array}{l}0 \widehat{\sqrt[n]{2}} \\
\bar{\Xi} \bar{d}\end{array}$ & 01 & 01 & 11 & $\begin{array}{l}r \hat{\sigma} \\
\infty \hat{0} \\
=\hat{d}\end{array}$ \\
\hline 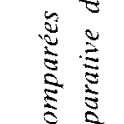 & & $\begin{array}{l}\text { 总㝘 } \\
\text { 点 } \\
\text { 营 }\end{array}$ & & 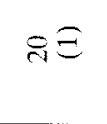 & $\cong \Xi$ & $\mathrm{d}=$ & $\approx \widehat{\Xi}$ & | 1 & নి \\
\hline$\frac{\tilde{3}}{5}$ & 总 & 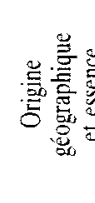 & & 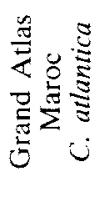 & 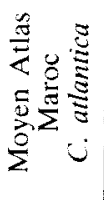 & 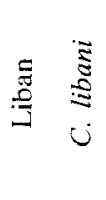 & 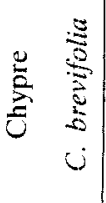 & 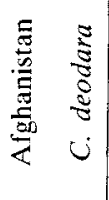 & 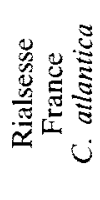 \\
\hline
\end{tabular}


TABLEAU 3

Densités comparées de Cedrobium laportei et de Cinara cedri sur diverses provenances de cèdres dans la plantation de Pellenc (Var).

Comparative densities of Cedrobium laportei and Cinara cedri on different cedar provenances in Pellenc's plantation (Var).

\begin{tabular}{|c|c|c|c|c|c|c|}
\hline \multirow{3}{*}{$\begin{array}{c}\text { Dates } \\
\begin{array}{c}\text { Origine } \\
\text { gćographique } \\
\text { et essence }\end{array}\end{array}$} & \multicolumn{3}{|c|}{ Juin 1981} & \multicolumn{3}{|c|}{ Juin 1985} \\
\hline & \multirow{2}{*}{$\begin{array}{l}\text { Nbre arbres } \\
\text { (Nbre prov.) }\end{array}$} & \multicolumn{2}{|c|}{$\begin{array}{c}\text { Densité moyenne/arbrc } \\
\text { (Ecart-type) }\end{array}$} & \multirow{2}{*}{$\begin{array}{l}\text { Nbre arbres } \\
\text { (nbre prov.) }\end{array}$} & \multicolumn{2}{|c|}{$\begin{array}{c}\text { Densité moyenne/arbre } \\
\text { (Ecart-type) }\end{array}$} \\
\hline & & C. laportei & C. cedri & & C. laportei & C. cedri \\
\hline $\begin{array}{l}\text { Grand Atlas } \\
\text { Maroc } \\
\text { C. atlantica }\end{array}$ & $\begin{array}{c}7 \\
(1)\end{array}$ & $\underline{0}$ & $\begin{array}{c}15,3 \\
(27,2)\end{array}$ & - & - & - \\
\hline $\begin{array}{c}\text { Moyen Atlas } \\
\text { Maroc } \\
\text { C. atlantica }\end{array}$ & $\begin{array}{r}102 \\
(8)\end{array}$ & $\begin{array}{c}4,6 \\
(46,5)\end{array}$ & $\begin{array}{c}8,3 \\
(34,8)\end{array}$ & $\begin{array}{l}10 \\
(1)\end{array}$ & $\begin{array}{c}173,2 \\
(111,8)\end{array}$ & $\begin{array}{c}450,4 \\
(597,7)\end{array}$ \\
\hline $\begin{array}{c}\mathrm{Rif} \\
\mathrm{Maroc} \\
\text { C. atlantica }\end{array}$ & $\begin{array}{l}41 \\
(2)\end{array}$ & $\begin{array}{c}7,4 \\
(32,3)\end{array}$ & $\begin{array}{c}1,7 \\
(8,0)\end{array}$ & - & - & - \\
\hline $\begin{array}{l}\text { Liban } \\
\text { C. libani }\end{array}$ & $\begin{array}{c}7 \\
(1)\end{array}$ & $\frac{0}{-}$ & $\underline{0}$ & $\begin{array}{l}20 \\
(2)\end{array}$ & $\frac{0}{-}$ & $\begin{array}{l}(169,1) \\
(309,9)\end{array}$ \\
\hline $\begin{array}{c}\text { Syrie } \\
\text { C. libani }\end{array}$ & - & - & - & $\begin{array}{l}10 \\
(1)\end{array}$ & $\underline{0}$ & $\begin{array}{l}(139,0) \\
(211,4)\end{array}$ \\
\hline $\begin{array}{l}\text { Turquie } \\
\text { C. libani }\end{array}$ & - & - & - & $\begin{array}{l}20 \\
(1)\end{array}$ & $\frac{0}{-}$ & $\begin{array}{c}67 \\
(108,2)\end{array}$ \\
\hline $\begin{array}{c}\text { Chypre } \\
\text { C. brevifolia }\end{array}$ & $\begin{array}{l}43 \\
(2)\end{array}$ & $\frac{0}{-}$ & $\begin{array}{c}0,2 \\
(1,5)\end{array}$ & $\begin{array}{l}10 \\
(1)\end{array}$ & $\underline{0}$ & $\begin{array}{c}247,6 \\
(374,2)\end{array}$ \\
\hline $\begin{array}{c}\text { Ventoux } \\
\text { France } \\
\text { C. atlantica }\end{array}$ & - & - & - & $\begin{array}{l}10 \\
(1)\end{array}$ & $\begin{array}{l}58,2 \\
(94,4)\end{array}$ & $\begin{array}{c}172,2 \\
(126,0)\end{array}$ \\
\hline $\begin{array}{l}\text { Rialsesse } \\
\text { France } \\
\text { C. atlantica }\end{array}$ & $\begin{array}{c}7 \\
(1)\end{array}$ & $\frac{0}{-}$ & 0 & - & $\overline{-}$ & - \\
\hline
\end{tabular}


TABLEAU 4

Densité comparée de Cinara cedri sur diverses provenances de cèdres

dans la plantation de Pellenc (Var) en 1985 ( $F=2,07$; ddl : inter pop. 7, intra pop. 72).

Comparative density of Cinara cedri on different cedar provenances in Pellenc's plantation (Var) in $1985(F=2,07$; df between : 7 , within: 72).

\begin{tabular}{|c|c|c|c|c|c|c|c|c|}
\hline \multirow{2}{*}{ Espèce } & \multirow{2}{*}{\multicolumn{3}{|c|}{ Provenance }} & \multirow{2}{*}{$\begin{array}{l}\text { Nombre } \\
\text { d'arbres }\end{array}$} & \multirow{2}{*}{$\begin{array}{c}\text { Densité } \\
\text { C. cedri } \\
\text { moy./arbre }\end{array}$} & \multicolumn{3}{|c|}{ Test de Duncan au seuil } \\
\hline & & & & & & $1 \%$ & $5 \%$ & $10 \%$ \\
\hline atla. & CZAD & (Col du Zad) & Maroc & 10 & 450 & & & \\
\hline liba. & HAJI & (H. Jibbé) & Liban & 10 & 285 & & & \\
\hline brev. & PAPH & (Paphos) & Chypre & 10 & 248 & & & \\
\hline atla. & VENT & (Ventoux) & France & 10 & 172 & & ــ & \\
\hline liba. & SYR & (Syrie) & Syrie & 10 & 139 & & & \\
\hline liba. & POZA & (Pozanti) & Turquie & 10 & 111 & & & \\
\hline liba. & BARO & (Barouk) & Liban & 10 & 53 & & & \\
\hline liba. & SUTL & (Sutlegen) & Turquie & 10 & 23 & & & \\
\hline
\end{tabular}

\subsection{Cedrobium laportei}

Les provenances libanaises, turques et syriennes de $C$. libani et celle, chypriote, de C. brevifolia n'ont jamais été infestées par le puceron. Pourtant, nous avons examiné 138 arbres de $C$. libani représentant 5 provenances et 125 arbres de $C$. brevifolia représentant 3 provenances. Dans les conditions des deux plantations, $C$. libani et $C$. brevifolia semblent donc bien se montrer « résistantes" à $C$. laportei. A l'inverse, les provenances de $C$. atlantica étaient très infestées, ce qui n'est pas une surprise. Les densités d'infestation atteignaient au Treps sur certaines d'entre elles : $360(06.80$, Tounfite, Maroc), 334 (06.81, Rialsesse, France), 142 (06.85, Tounfite, Maroc), à Pellenc 173 (06.85, Col du zad, Maroc). Enfin quelques petites colonies ont été trouvées sur de jeunes $C$. deodara au Treps en 1985.

Une analyse plus fine des densités d'infestation relatives des provenances de $C$. atlantica n'a pu être faite à Pellenc en 1981 car elles étaient encore trop faibles (4,61 \pm 9 pucerons par arbre). Une année auparavant, on pouvait à peine y déceler la présence de $C$. laportei. Ceci résultait du fait que les premiers vieux cèdres à partir desquels s'est opéré la colonisation par le puceron de la plantation, en sont distants de plus de $5 \mathrm{~km}$. En 1985 en revanche, $C$. laportei était abondant $(173,2 \pm 69,3$ pucerons par arbre), mais les observations n'ont malheureusement porté cette année-là que sur deux provenances.

L'analyse a donc été faite dans la plantation du Treps, contaminée plus précocement, à partir d'une trentaine d'arbres cinquantenaires, isolés, situés dans la direction du vent dominant, à quelques centaines de mètres seulement. Des différences significatives entre 8 provenances infestées ont été mises en évidence en 1980, puis à nouveau en 1985 (tabl. 5). Dans les deux cas, ce sont les provenances TOUN (Tounfite, Maroc, Haut-Atlas) et SEHE (Seheb, Maroc, Moyen-Atlas) qui ont été les plus attaquées. En 1981, en revanche, tant en juin qu'en octobre, aucune différence significative (même au seuil 25 p. 100) entre les mêmes provenances n'a été observée. 


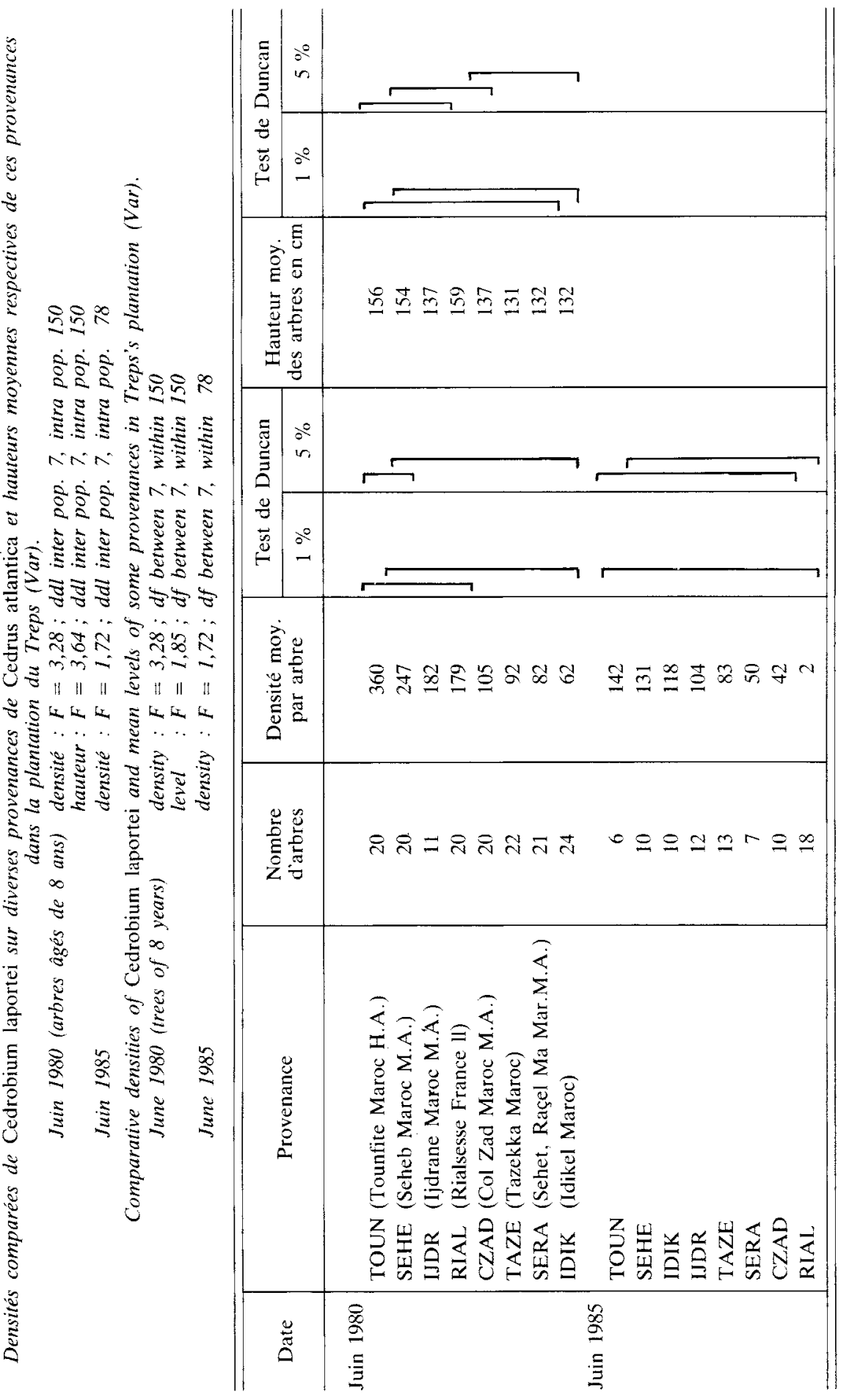


Ce dernier résultat, apparemment en contradiction avec les autres, s'explique par les conditions climatiques : au printemps 1981, dès le mois de janvier, la température s'est maintenue nettement au-dessus de la normale, ce qui a permis à $C$. laportei de se multiplier très précocement, si bien qu'au mois de juin l'insecte se trouvait avoir colonisé de façon assez uniforme l'ensemble du peuplement. Au mois d'octobre, les densités d'infestations étaient trop faibles (en moyenne de $11,5 \pm 2,9$ ). A l'automne, les pullulations sont au contraire toujours limitées par les conditions climatiques.

Par ailleurs, au mois de juin en 1980, des différences hautement significatives de hauteur existaient entre les provenances (tabl. 5). De plus, la densité d'infestation moyenne par provenance $(\mathrm{Y})$ était liée à la taille moyenne de chaque provenance $(\mathrm{X})$ : $\mathrm{Y}=6,8 * \mathrm{X}-803,9$ avec $: \mathrm{r}=0,81, \mathrm{~F}=39,6$ (1 et 6 d.d.l.), $\mathrm{P}=2,5 \mathrm{p} .100$. Cette relation est encore meilleure entre les individus quelle que soit leur origine géographique, en utilisant comme variable explicative la différence de la hauteur de l'arbre $\left(\mathrm{X}^{\prime}\right)$ soit par rapport à l'arbre moyen de l'ensemble des individus: $\mathrm{Y}^{\prime}=2,5 * \mathrm{X}^{\prime}+158,6$, $r=0,28, F=13,6$ ( 1 et 156 d.d.l.), $P=0,5$ p. 100, soit par rapport à l'arbre moyen de chaque parcelle: $\mathrm{Y}^{\prime}=2,6 * \mathrm{X}^{\prime}+158,6, \quad \mathrm{r}=0,22, \mathrm{~F}=7,8, \mathrm{P}=0,5 \mathrm{p}$. 100 . Plusieurs hypothèses sont possibles. La plus vraisemblable est que dans ce peuplement en cours de colonisation, les pucerons se soient développés sur les arbres les plus hauts de chaque parcelle, sur lesquels ils ont été transportés par le vent. En juin 1981, les corrélations précédentes, aussi bien entre les provenances qu'entre les individus ne sont significatives qu'au seuil de 25 p. 100 . Comme nous l'avons déjà signalé, cette année-là, très tôt des individus ailés se sont portés sur les autres arbres y compris sur ceux de plus petite taille.

\subsection{Possibilités d'infestations artificielles de C. laportei chez les cèdres}

\subsection{Comparaison des espèces : C. atlantica, C. deodara, C. brevifolia}

\subsection{En serre}

Cet essai devait vérifier que $C$. laportei ne pouvait ni se fixer, ni se multiplier sur C. brevifolia. Dans ce but, 5 arbres supplémentaires de cette même espèce ont été infestés en même temps aux Vignères (tabl. 1). Ce dernier résultat n'a pas été reporté sur le graphique la, un autre essai ayant été réalisé dans les conditions naturelles.

Le 16.05.1983 $(j+3)$, sur $C$. brevifolia, en serre, tous les pucerons sont morts alors qu'aux Vignères, avant de disparaître $(j+4)$, quelques-uns sont retrouvés vivants sur les pousses de l'année précédente, sur $C$. deodara, une cinquantaine se sont fixés, sur $C$. atlantica, ils ont donné naissance à de petites colonies de 5-10 individus. Le $20.05(j+7)$, sur $C$. deodara le nombre de pucerons est resté stationnaire, sur Cedrus atlantica, en revanche, il a fortement augmenté. Par la suite, C. laportei disparaît progressivement sur ces deux espèces. Cette disparition est la conséquence de l'action des températures qui atteignent puis dépassent le seuil thermique supérieur de développement de l'insecte (FABre \& Rabasse, 1987). En serre, ce seuil est atteint plus tôt que dans les conditions naturelles. Quoi qu'il en soit, on a dénombré au total: 3164 pucerons sur la provenance de $C$. atlantica, 385 sur la provenance Himalayenne de $C$. deodara. 


\subsection{Dans les conditions naturelles}

Les résultats sont consignés sur la figure $1 \mathrm{~b}$. Le $30.04 .1985(j+1)$, de 10 à 20 pucerons sont fixés sur les 3 espèces de cèdres y compris $C$. brevifolia. Deux jours plus tard sur cette dernière espèce on en retrouvait encore 7 , mais le $06.05(j+7)$, ils avaient disparu ou étaient retrouvés morts dans les manchons. En revanche, on dénombrait sur chaque arbre de 105 à 360 pucerons sur $C$. atlantica, de 102 à 506 pucerons sur $C$. deodara. Puis (fig. 1b), sur ces deux espèces, $C$. laportei s'est fortement multiplié jusqu'au $10.06(j+42)$. Sur un arbre il y avait 3000 pucerons, une forte production de miellat sur lequel s'est développée de la fumagine. La température devenant trop élevée, on a enlevé les manchons, mais des prédateurs qui étaient présents sur d'autres arbres, hors essai, ont anéanti progressivement les colonies.
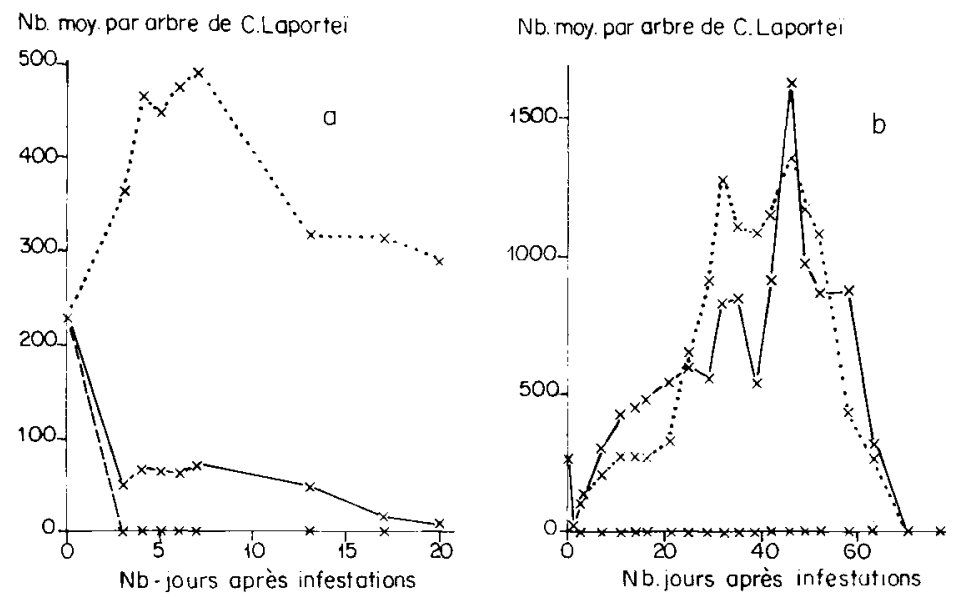

FIG. 1

Infestations artificielles de Cedrobium laportei de trois espèces de Cẹdre: C. atlantica, C. deodara,

C. brevifolia, d'une part en serre (a), d'autre part dans les conditions naturelles $(b)$.

Artificial infestations of tree cedar species: C. atlantica, C. deodara, C. brevifolia

to Cedrobium laportei artificial infestations, in greenhouse (a) and in natural conditions (b).

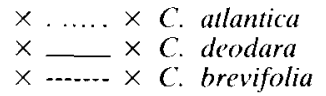

\subsection{Comparaison C. atlantica, C. brevifolia, C. libani}

A Pellenc, le $27.06 .1985(j+6)$, les 1000 pucerons installés sur $C$. brevifolia ont disparu ou sont retrouvés morts. 21 pucerons $(0,4 \mathrm{p} .100$ de la population initiale $)$ se sont fixés sur 2 provenances turques de $C$. libani : 3 sur POZA et 18 sur SULT, les autres : 2 provenances libanaises, 1 syrienne étant indemnes. En revanche, 496 pucerons, soit 25 p. 100 de la population initiale se sont fixés sur $C$. atlantica représentée ici par une provenance du Moyen Atlas (CZAD) sur laquelle on a dénombré 354 pucerons et par une provenance française (VENT). Malheureusement, cette expérience a été réalisée trop tard en saison au moment où s'installaient les conditions climatiques estivales, de telle sorte que les pucerons fixés ne se sont pas multipliés et ils ont 
disparu le $10.07(\mathrm{j}+10)$ sur $C$. libani, le $11.07(\mathrm{j}+11)$ sur $C$. atlantica. Dans cette plantation, la population naturelle de $C$. laportei suivie sur $C$. atlantica du Ventoux a également disparu le 21.07.

La fixation de $C$. laportei sur $C$. brevifolia n'a pu être observée, mais signalons toutefois que nous avons trouvé quelques individus ailés qui venaient vraisemblablement de la parcelle, ce qui montre que cette essence n'est pas totalement répulsive pour le puceron.

\subsection{Comparaison de quelques provenances françaises de Cèdres et $\mathrm{C}$. deodara}

Pour ce dernier essai, plus marginal, nous avons choisi 1 provenance indienne et 4 provenances artificielles françaises. Celle du Ventoux est originaire d'Algérie (MaURY, 1960 ; Тотн, 1970-1972 ; Arbez et al., 1978). Celles de Villelaure (84) et de SaintMichel l'Observatoire (04) viennent probablement du Liban, mais pour cette dernière on sait qu'elle a été mélangée avec le cèdre de l'Atlas si bien qu'à l'heure actuelle il existe des individus du type «Liban» et dautres du type «Atlas». Enfin, celle de Marcilly appartient à $C$. atlantica.
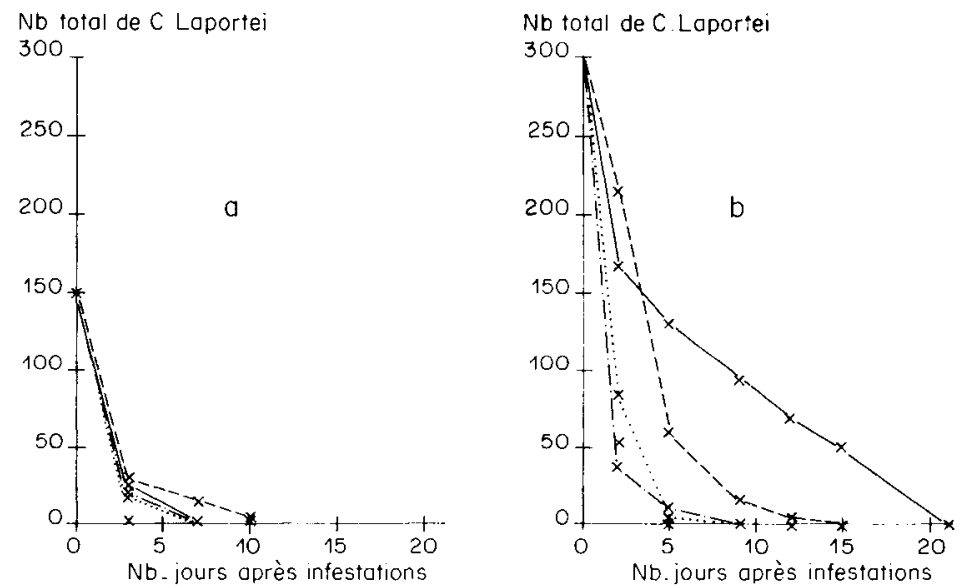

FIG. 2

Infestations artificielles de Cedrobium laportei ( $a$ : infestations du 21-05-1985,

$b$ : infestations du 05-06-1985), de quatre provenances artificielles françaises de Cèdre et de C. deodara, dans les conditions naturelles.

Artificial infestations of four cedar artificial french provenances and $\mathrm{C}$. deodara to artificial Cedrobium laportei's infestations (a: first infestations of 21-05-1985, $b$ : second infestations of 05-06-1985), in natural conditions.

$\times$ _ $\times$ atlantica : Marcilly, Forêt domaniale des Solanes de Nore (11).

$\times$-..-- $\times$ atlantica: Ventoux, Forêt communale de Bédoin' (84).

$\times \ldots \ldots \times$ Type "Liban »: $2^{e}$ génération, Saint-Michel-l'Observatoire (04)

$\times \quad \times$ Type « Liban $":$ Villelaure $(84)$.

$\times$ - $x$ deodara : HIMA, Inde.

Une première infestation a été faite le 21.05 .85 avec 50 pucerons par arbre. Le $24.05(j+3)$ quelques pucerons seulement se sont fixés (fig. 2a), puis ils disparurent rapidement sauf sur la provenance du Ventoux et celle de Marcilly. Les résultats sont 
insuffisants et nous effectuons une deuxième infestation le 05.06 .85 , cette fois avec 100 individus par arbre (fig. 2b). Les pucerons disparaissent lé : 10.06 (Villelaure), 14.06 (Saint-Michel et $C$. deodara), 20.06 (Ventoux), 26.06 (Marcilly). En définitive C. laportei ne s'est pas multiplié, mais le nombre total de pucerons dénombrés permet de donner le classement suivant :

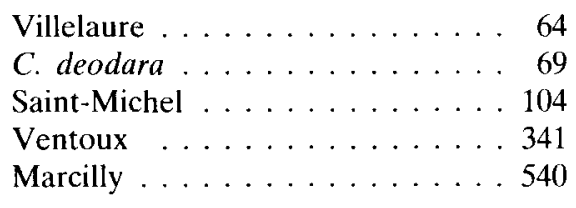

Il est conforme aux résultats précédents : les 2 provenances de $C$. atlantica ont été les plus infestées, en revanche, celles du «type Liban»le sont beaucoup moins tout comme celle de $C$. deodara.

\section{Conclusions}

- Cinara cedri infeste spontanément les 4 espèces de cèdre. Cependant, $C$. deodara dont l'aire naturelle est très vaste n'est représenté dans les plantations comparatives que par deux provenances. Par ailleurs, nous avons mis en évidence des différences significatives de densité d'infestation entre certaines provenances de $C$. atlantica. Avant d'être prises en considération, elles demanderaient à être confirmées et expliquées. Il faudrait aussi prendre en compte l'impact de ce puceron sur les cèdres, d'autant plus qu'il semble beaucoup plus faible que pour l'autre espèce (FABRE, 1982). Quoi qu'il en soit, ces résultats s'accordent avec le fait que $C$. cedri ayant été trouvé dans une partie de l'aire naturelle des cèdres, rien ne s'oppose à sa découverte en Algérie sur $C$. atlantica, à Chypre sur $C$. brevifolia, en Afghanistan sur $C$. deodara.

C. atlantica est l'espèce la plus infestée par $C$. laportei. Cependant, entre quelquesunes de ses provenances, des différences ont été mises en évidence en 1980 puis en 1985. En réalité, les colonies se sont développées dans chaque placette sur la pousse terminale des arbres les plus hauts quelle que soit leur origine géographique, peut-être à partir d'individus transportés par le vent. Les relevés devraient être poursuivis dans cette même plantation lorsqu'elle ne sera plus en situation de colonisation. D'autres devraient être effectués dans des reboisements ne comportant qu'une seule provenance. De plus, les différences ne sont perceptibles qu'au début du printemps, sans doute avant l'apparition des ailés qui se dispersent activement. Enfin dans ce type d'étude, il est indispensable pour préciser la « sensibilité » des provenances à $C$. laportei, d'étudier leur degré "d'infestabilité », mais aussi de le relier au seuil de nuisibilité de l'insecte, ce qui n’a pas été fait dans cette étude préliminaire. $C$. deodara, représenté par une seule provenance afghane était également infesté. Par contre, $C$. laportei n'a pas été observé, ni sur $C$. brevifolia, ni sur $C$. libani. Ces derniers résultats sont en accord avec le fait que $C$. laportei n'a pas été trouvé lors, des prospections faites en Turquie, en Syrie et au Liban par Remaudière.

L'utilisation de la technique des infestations artificielles était à priori séduisante, mais il faut disposer de 200 à $300 \mathrm{C}$. laportei par infestation. Pour cela, il est indispensable d'attendre le développement de ses populations spontanées qui s'effectue 
généralement sur une courte période ( 1 à 2 mois). En conséquence, les expériences ont été effectuées trop tard en saison ct nous avons enregistré le plus souvent la disparition des pucerons. Dans un seul cas, grâce à un élevage conduit en serre sur $C$. atlantica, nous avons pu effectuer les infestations plus tôt (le 29.04.85) et observer la multiplication des pucerons.

Quoi qu'il en soit, les infestations artificielles confirment les résultats obtenus à partir des infestations spontanées, tout en les nuançant dans certains cas. Ainsi, $C$. atlantica s'est toujours montré l'espèce la plus «infestable » y compris la provenance artificielle française du Ventoux (originaire d'Algérie) et celle du Minervois (Marcilly). C. brevifolia était bien l'espèce la plus « résistante ", mais C. laportei peut s'y fixer pendant 2 à 3 jours. $C$. libani se rapproche nettement de $C$. brevifolia mais les pucerons peuvent s'y fixer plus facilement et s'y maintenir pendant un certain temps. Enfin, $C$. deodara représentée par une seule provenance indienne a été faiblement infestée tout comme $C$. libani. Dans un cas elle a été aussi infestée que $C$. atlantica.

A l'heure actuelle, dans le sud de la France, l'extension du cèdre se fait exclusivement au moyen de $C$. atlantica. Les provenances artificielles françaises ont une meilleure croissance en plantation comparative (VEYRET, 1984) et on peut récolter facilement des graines. Cependant, à la lumière de ces résultats préliminaires, l'utilisation de $C$. libani et peut-être dans certains cas de $C$. brevifolia serait souhaitable pour éviter les risques de pullulation de $C$. laportei. Ils sont importants à basse altitude (Fabre, 1982). Dans un reboisement de 26 ans situé à $330 \mathrm{~m}$ d'altitude, au Petit Lubéron dans la zone de chêne kermès où nous suivons depuis 1975 l'évolution des niveaux de population des pucerons, 10 p. 100 des arbres sont morts, chaque fois après une très forte multiplication de $C$. laportei.

\section{Reçu le 5 août 1987.}

Accepté le 7 novembre 1987.

\section{Remerciements}

Nous remercions bien vivement $M$. Arbez et P. Frrrandez (Amélioration des arbres forestiers) qui ont mis à notre disposition le dispositif des plantations comparatives de cèdre. C'est également P. Ferrandez qui nous a fourni les plants pour réaliser nos propres petites plantations. Nous remercions J. TOTH de la station de Sylviculture méditerranécnne qui nous a permis de réaliser des infestations dans la plantation des Vignères. Merci à P. Dumerle ct à D. Schivfster qui ont revu le manuscrit.

\section{Summary}

Comparative susceptibility of the genus Cedrus to aphids' infestations : Cedrobium laportei Remaudière and Cinara cedri Mimeur (Homoptera, Lachnidae).

This publication brings some precisions on the susceptibility of some provenances of : Cedrus atlantica Manetti, C. libani Loudon, C. brevifolia Henry. C. deodara Loudon (only two provenances), to aphids' infestations : Cedrobium laportei and Cinara cedri. 
We have estimated the natural infestations' densities of insects in two comparative plantations cedars' provenances in 1980, 1981, 1985. We also have conducted artificial infestations of Cedrobium laportei in a greenhouse and in natural conditions between some provenances of : Cedrus atlantica, C. brevifolia, $C$. deodara. Another test included provenances of C. libani. A last test was made on different artificial french provenances and on other one of $C$. deodara.

In natural conditions Cinara cedri can infest the four cedars' spccies. This aphid can be found, where it has not been yet discovered: Cyprus, Afghanistan, Algérie... On the other hand, the genus Cedrus offers important differences of susceptibility to Cedrobium laporteis infestations. Cedrus atlantica can be easily attacked; it's not a surprise. Cedrus deodara can be also attacked. On the contrary, Cedrus brevifolia and C. libani are not attacked by Cedrobium laportei and secm "resistant $»$ in natural conditions. Sometimes differences of susceptibility can be found between some provenances for the two species of aphids. In this case in each experimental plot $C$. laportei have infested the higher trees

Artificial infestations confirm the results for $C$. laportei as well as they give nuances in some cases. Indeed, Atlas cedar was always the more susceptible species, even french artificial provenances of Ventoux and Marcilly. The settlement of aphids was very difficult or impossible on Cedrus brevifolia, but it was observed quite regulary on $C$. libani. The provenance of $C$. deodara is more or less susceptible.

Key words: Cedar, Cedrus atlantica, Cedrus libani, Cedrus brevifolia, Cedrus deodara, Homoptera, Lachnidac, Cedrobium laportei, Cinara cedri, plant resistance, host specificity, susceptibility.

\section{Références bibliographiques}

ABi-Salen B., 1978. Etude phytodynamique et écologique des peuplements sylvatiques du Luberon. Signification bioclimatique et essai de cartographie dynamique. Thèse Doct. Sci. Université de droit d'économie et des Sciences. Faculté des Sciences et Techniques Saint-Jérome.

Achunl A., Akabli O., Barbero M., Benabid A., M'Hirit A., Peyre C., Quezel P., RivasMartinez S., 1980. A propos de la valeur bioclimatique et dynamique de quelques essences forestières au Maroc. Ecol. Mediterr., 5, 211-249.

Anonyme, 1975. Description du Réseau des plantations comparatives de provenances de cèdres. Document interne INRA, Station d'Amélioration des Arbres forestiers, Bordeaux, $40 \mathrm{p}$.

Arbez M.. Ferrandez P., Uyar M., 1978. Contribution à l'étude de la variabilité géographique des cèdres. Ann. Sci. forest., 35 (4), 265-284.

Aussenac G., 1983. Le cèdre, essai d'interprétation bioclimatique et écophysiologique. $C . R$. Colloque de Bioclimatologie méditerranéenne, 18-20 mai 1983, 4, 8.

BeAls E.W., 1965. The remnant cedar Forest of Lebanon. J. Ecol., 53, 679-694.

Carter C.I., Maslen N.H., 1982. Conifer lachnids in Britain. Forest. Comm. Bull., 58. 1-75.

Covassı M., 1971. Osscrvazioni preliminari sulla presenza in Italia di un afide nocivo ai Cedri : Cedrobium laportei Remaud. (Homoptera, Aphidoïdea, Lachnidae). Redia, 52, 641-652.

Covassi M., Binazzi A., 1974. Note corologiche e morphologiche sulla Cinara cedri MIM. in Italia (Homoptera Aphidoïdea Lachnidae). Redia, 55, 331-344.

Emonnot P., Gayraud Y., Lecl.ant F., Remaudir̀rf G., 1967. Sur la présence en France de Cedrobium laportei Remaudière, puceron nuisible au cèdre. C.R. Acad. Agric. Fr., 53, 966971 .

Fabre J.P., 1976. Sur la présence en France de Cinara cedri (Mimeur) puceron nuisible au cèdre. C.R. Acad. Agric. Fr., 2 juin 1976, 771-775.

FABRE J.P., 1982. Le problème des pucerons du cèdre. Dégâts et méthodes de lutte. C.R. Journées d'Etudes et d'Informations "Pucerons des cultures", Paris, 2-3 mars 1981. - Association de coordination technique agricole, Paris, 331-335.

Fabre J.P., Rabasse J.M., 1987. Introduction dans le sud-est de la France d'un parasite : Pauesia cedrobii Stary et Leclant (Hym. Aphidiidae) du puceron: Cedrobium laportei Remaudière (Hom., Lachnidae) du cèdre de l'Atlas : Cedrus atlantica Manetti. Entomophaga, 32 (2), 127 141 . 
Maury R., 1960. Le reboisement de la Forêt de Bédoin et son enseignement. Ann. Ecole Nat. E. F. Nancy, 17 (1), 119-153.

Mimeur J.M., 1935. Aphididae du Maroc (7 note). Bull. Soc. Sci. Nat. Maroc, 15 (3), $251-258$.

Notario A., Cadierno D., Mujares A., 1978. Presencia en Hoyo de Manzanares (Madrid) de un puegon que ataca a los Cedros Cinara cedri Mimeur. Ann. Inst. Nacional de Investigaciones Agrarias, 8, 59-64.

Pradal F., 1979. Variabilité génétique et écophysiologique du cèdre. Mémoire de fin d'études à I'E.N.I.T.E.F., 89 p.

Quezel. P. \& Barbero M., 1985. Carte de la végétation potentielle de la région méditerranéenne. C.N.R.S. Edt. Paris, 69 p.

Quezel. P., Pamukcuoglu A., 1973. Contribution à l'étude phytosociologique et bioclimatique de quelques groupements forestiers du Taurus. Feddes Reperiorium, 84 (3), 185-229.

Rfmaudière G., 1954. Les Cinarini (Hym. Aphidoidea, Lachnidae) du cèdre en Afrique du Nord. Rev. Path. vég. Ent. agr. France, 33, 115-122.

Roberti D.. 1976. Sulla presenza del Cedrobium laportei Rem. (Homoptera, Aphidoidea, Lachnidae) nell'Italia meridionale. Entomologica, 12, 57-62.

Rossem G. van, Bund C.F. van DE, Burger H.C., 1977. Bijzondere aantastingen door inskten in 1976. Entomologische Bericht., 37, 97-98.

ShandS W.A., Simpson G.W.. 1955. Survey methods : populations of potato-infesting aphids an aphids eggs on primary host in Maine. Coop. econ. Insect., Rep., 23-30.

Tотн J., 1970-1972. Historique du cèdre sur le Mont-Ventoux Bull. Soc. Sc. nat., Vaucluse, 51-75.

Toтн J., 1982-1984. Quelques éléments nouveaux pour mieux situer et caractériser le Cèdre de l'Himalaya vis-à-vis du Cèdre de l'Atlas et du Cèdre du Liban en France méridionale. Bull. Soc. Sci. nat. Vaucluse, 41-49.

Tutay N. \& Remaudière, G., 1964. Première contribution au catalogue des Aphididae (Hom.), de la Turquic. Rev. Path. vég. Ent. agric. France, 43 (4), 243-278.

Veyret P., 1984. Variabilité génétique des provenances naturelles et artificielles de cèdres. Mémoire de stage de B.T.S., option «Forêt », Ecole Forestière de Meymac, 43 p. 